\title{
Some Like It Cold: Postpolypectomy Delayed Hemorrhage in Anticoagulated Patients
}

\author{
Yu-Hsi Hsieh ${ }^{1,2}$. Malcolm Koo Ko $^{3,4}$ \\ Published online: 28 February 2019 \\ ○) Springer Science+Business Media, LLC, part of Springer Nature 2019
}

Current estimates suggest that colonoscopic polypectomy prevents $80 \%$ of interval colon cancers. Conventionally, polypectomy performed with electrocautery is termed 'hot snare' polypectomy (HSP), which carries a small but significant complication rate, including immediate and delayed postpolypectomy bleeding (DPPB), transmural thermal injury, and perforation. Cold snare polypectomy (CSP) has been recently recommended as the method of choice for removing colon polyps $\leq 9 \mathrm{~mm}$ [1], which comprise the majority of the polyps identified and removed during colonoscopy. Without the need for electrocautery and low risk of transecting the muscularis propria, it almost eliminates the risk of transmural thermal injury and perforation. Bleeding immediately after polypectomy, especially the capillary bleeding following the removal of small colonic polyps, generally stops spontaneously, or is easily treated with endoscopic hemostasis, such as clipping, and therefore, is of less concern for endoscopists. Therefore, DPPB has become the major complication of concern following CSP.

The risk of DPPB increases with the size of resected polyps and in patients receiving antithrombotic therapy [2]. In a recent report, the risk of DPPB requiring endoscopic hemostasis is low after HSP removal of 4-9-mm adenomatous polyps $(0.5 \%, 2$ of 402 polyps $)$ in patients not using antithrombotic therapy [3]. Yet, the risk was markedly increased in patients taking antithrombotic drugs [4], and

Yu-Hsi Hsieh

hsieh.yuhsi@msa.hinet.net

1 Division of Gastroenterology, Department of Medicine, Dalin Tzu Chi Hospital, Buddhist Tzu Chi Medical Foundation, 2 Minsheng Road, Dalin, Chiayi 62247, Taiwan

2 School of Medicine, Buddhist Tzu Chi University, Hualien, Taiwan

3 Graduate Institute of Long-Term Care, Tzu Chi University of Science and Technology, Hualien, Taiwan

4 Dalla Lana School of Public Health, University of Toronto, Toronto, ON, Canada the combined use of multiple antithrombotic agents puts patients at an even higher risk [5]. Current practice guidelines consider polypectomy a high-risk procedure in patients requiring long-term antithrombotic agents, recommending temporary withdrawal of these agents prior to polypectomy, increasing the risk of thrombotic complications [6]. In a recent study conducted in a population of anticoagulated patients, the risk of DPPB was substantially reduced from $14 \%$ in those using HSP to $0 \%$ in those using CSP despite ongoing use of anticoagulants [4], a benefit possibly attributable to less submucosal arterial injury [4]. Histological studies of ulcers 1 day after polypectomy also showed that the diameter increased by $29 \%$ with HSP, but decreased by $25 \%$ with CSP [7]. Nonetheless, the safety of CSP for patients using antithrombotic drugs is not well established, especially for those taking multiple antithrombotics.

In this issue of Digestive Diseases and Sciences, Arimoto et al. retrospectively reviewed the records of 501 consecutive patients bearing 1177 polyps $\leq 10 \mathrm{~mm}$ undergoing CSP, with 266 polyps in patients taking anticoagulant therapy and 911 polyps in patients who did not take antithrombotics [8]. Antithrombotic agents were continued even on the day of CSP in all patients in the antithrombotic group. The primary outcome, defined as DPPB within 2 weeks after polypectomy, did not occur in any case, irrespective of using antithrombotics or not. The use of antithrombotics was identified as a significant risk factor for immediate bleeding, which occurred in 63 (5.4\%) cases and was readily controlled without any significant clinical consequences. The finding of no cases of DPPB is compatible with several available studies of patients taking antithrombotics without discontinuation prior to polypectomy (Table 1), with rates of DPPB ranging from 0 to $1.2 \%[4,9,10]$, lending support to the safety of CSP.

Several nuances in this study have to be taken into account when interpreting its results. All polyp resections were performed with a dedicated cold snare, SnareMaster Plus (Olympus), which has a coated thin wire $(0.3 \mathrm{~mm})$ and 


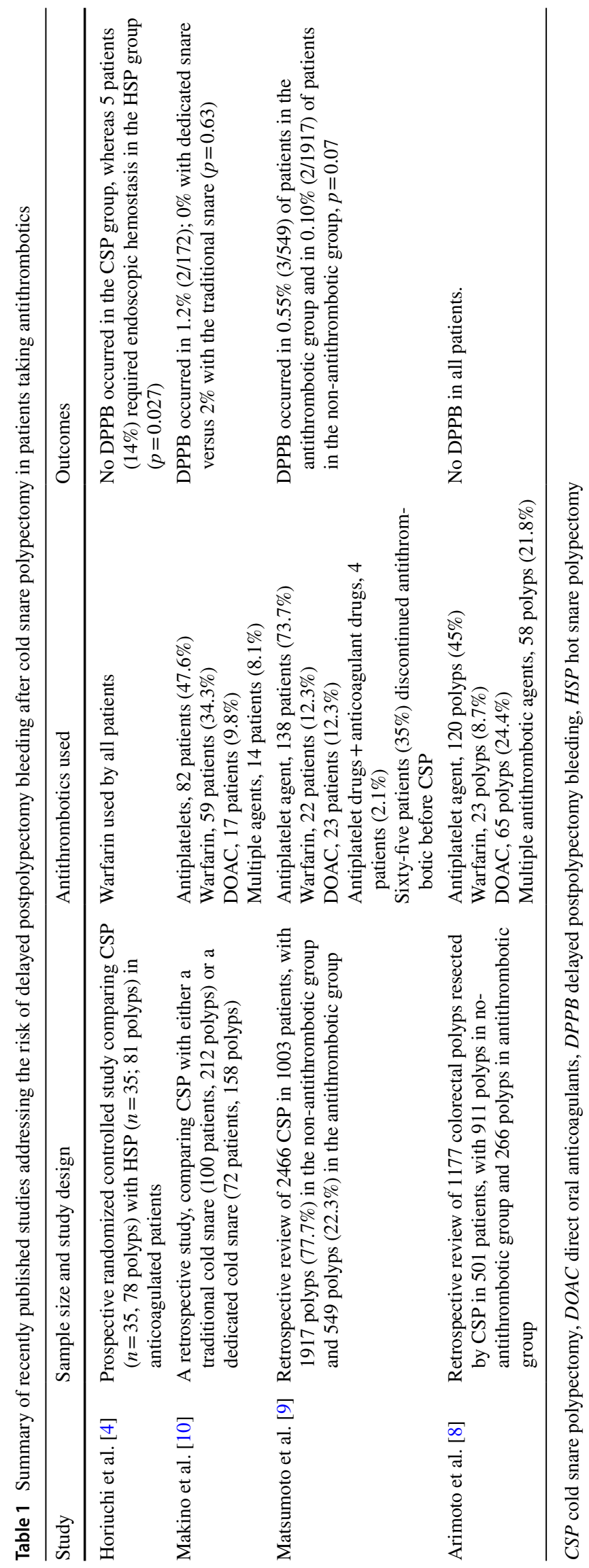


a distinctive shape that was stated to cause less injury to submucosal arteries than a traditional cold snare [10]. Although prophylactic clipping was performed in $13.9 \%$ of patients in the antithrombotic group, its effects on DPPB after CSP were unknown. A transparent cap mounted to the tip of the colonoscope was used in all cases in the study, which might facilitate polypectomy. It is, however, not a routine practice in many endoscopic units. The use of a dedicated snare, clips, and a transparent cap might all help to achieve the zero DPPB rate in the current study. Furthermore, the definition of bleeding should be factored in as well. In the current study, DPPB was defined as a fall in the hemoglobin level by at least $2 \mathrm{~g} / \mathrm{dL}$ below the most recent preoperative level, or necessitation of endoscopic hemostasis and/or blood transfusion, and/or massive melena, that is to say, hemorrhage of a lesser degree would have gone unreported. Also, the need for endoscopic hemostasis and/or blood transfusion was at the discretion of the endoscopist, and the definition of massive melena was left open to debate.

The current study also has a number of strengths. First, it had a relatively large sample size. Second, patients in the antithrombotic group received numerous antithrombotics, including drugs inhibiting platelet function, traditional vitamin $\mathrm{K}$ antagonists, and direct oral anticoagulants (DOACs), with $21.8 \%$ of subjects using multiple antithrombotic agents, which might carry an even higher risk of bleeding following polypectomy. As recommended by current endoscopic guidelines for the management of antithrombotic agents [6], most institutions temporarily interrupt antithrombotic before polypectomy, in contrast to the current study that provides support for polypectomy without withdrawing antithrombotic agents, a new and safer approach. Nevertheless, the study also has several limitations. It was a retrospective analysis of patients from a single hospital. The mean size of the polyps, $4.54 \mathrm{~mm}$ (SD $1.79 \mathrm{~mm}$ ), was relatively small compared with other studies using CSP for removing subcentimeter polyps $[4,10]$.

In conclusion, the authors of the current study are to be commended for cogently demonstrating the remarkable safety of CSP by showing no DPPB after $>1000$ CSPs, with some patients taking multiple antithrombotic agents. Nevertheless, the results are not conclusive with several issues raised. Since the risk of bleeding increases with the size of the polyp, a more stringent test for CSP lies in the resection of larger subcentimeter polyps, such as those 6-10 $\mathrm{mm}$, which were underrepresented in the current study. The impact of the preventive use of clips on DPPB warrants further investigation. A head-to-head comparison of a conventional snare and a dedicated snare would also be informative. In the end, if randomized, controlled trials support the conclusion that CSP is a safe approach carrying a very low risk of DPPB in anticoagulated patients, the vast majority of patients undergoing colonoscopy would not have to stop taking antithrombotic agents before colonoscopy and be subject to the increased risk of thromboembolic events. In spite of that, this approach also has its downsides. When polyps $\geq 1 \mathrm{~cm}$ are found during colonoscopy in patients taking antithrombotics without interruption, the patient will have to be rescheduled and go through bowel preparation and colonoscopy again after stopping the antithrombotics, with a consequent marked increase in cost, time, risk, and inconvenience. Balanced against the apparent safety and convenience of CSP for small polyps in anticoagulated patients, the cost-effectiveness of such a practice should be evaluated.

\section{References}

1. Ferlitsch M, Moss A, Hassan C, et al. Colorectal polypectomy and endoscopic mucosal resection (EMR): European Society of Gastrointestinal Endoscopy (ESGE) Clinical Guideline. Endoscopy. 2017;49:270-297.

2. Repici A, Hassan C, Vitetta E, et al. Safety of cold polypectomy for $<10 \mathrm{~mm}$ polyps at colonoscopy: a prospective multicenter study. Endoscopy. 2012;44:27-31.

3. Kawamura T, Takeuchi Y, Asai S, et al. A comparison of the resection rate for cold and hot snare polypectomy for 4-9 mm colorectal polyps: a multicentre randomised controlled trial (CRESCENT study). Gut. 2018;67:1950-1957.

4. Horiuchi A, Nakayama Y, Kajiyama M, Tanaka N, Sano K, Graham DY. Removal of small colorectal polyps in anticoagulated patients: a prospective randomized comparison of cold snare and conventional polypectomy. Gastrointest Endosc. 2014;79:417-423

5. Kishida Y, Hotta K, Imai K, et al. Risk analysis of colorectal post-polypectomy bleeding due to antithrombotic agent. Digestion. 2019;99:148-156.

6. Anderson MA, Ben-Menachem T, Gan SI, et al. Management of antithrombotic agents for endoscopic procedures. Gastrointest Endosc. 2009;70:1060-1070.

7. Suzuki S, Gotoda T, Kusano C, et al. Width and depth of resection for small colorectal polyps: hot versus cold snare polypectomy. Gastrointest Endosc. 2018;87:1095-1103.

8. Arimoto J, Chiba H, Ashikari K, et al. Safety of cold snare polypectomy in patients receiving treatment with antithrombotic agents. Dig Dis Sci. (Epub ahead of print). https://doi.org/10.1007/ s10620-019-5469-1.

9. Matsumoto M, Yoshii S, Shigesawa T, et al. Safety of cold polypectomy for colorectal polyps in patients on antithrombotic medication. Digestion. 2018;97:76-81.

10. Makino T, Horiuchi A, Kajiyama M, Tanaka N, Sano K, Maetani I. Delayed bleeding following cold snare polypectomy for small colorectal polyps in patients taking antithrombotic agents. J Clin Gastroenterol. 2018;52:502-507.

Publisher's Note Springer Nature remains neutral with regard to jurisdictional claims in published maps and institutional affiliations. 